\title{
Tissue-engineered augmentation of a rotator cuff tendon using a reconstituted collagen scaffold: a histological evaluation in sheep
}

\author{
Craig Van Kampen ${ }^{1}$ \\ Steven Arnoczky² \\ Patrick Parks ${ }^{3}$ \\ Eileen Hackett ${ }^{4}$ \\ Dana RuehIman 4 \\ Anthony Turner ${ }^{4}$ \\ Theodore Schlegel ${ }^{5}$
}

1 Rotation Medical, Inc., Plymouth, Minnesota, USA

2 Laboratory for Comparative Orthopaedic Research, College of Veterinary Medicine, Michigan State University, East Lansing, Michigan, USA

3 Histopathometrics, LLC, Mendota Heights, Minnesota, USA

4 Small Ruminant Comparative Orthopedic Laboratory, Department of Clinical Sciences, Colorado State University, Ft. Collins, Colorado, USA

5 Steadman Hawkins Clinic Denver, Greenwood Village, Colorado, USA

Corresponding author:

Steven Arnoczky

Laboratory for Comparative Orthopaedic Research

Michigan State University

East Lansing, MI 48824, USA

E-mail: arnoczky@cvm.msu.edu

\section{Summary}

To determine if an absorbable collagen scaffold of high porosity would allow rapid tissue ingrowth and permit the functional maturation and alignment of tendon-like tissue, scaffolds were sutured to the superficial surface of the infraspinatus tendons of adult sheep. Histology demonstrated complete ingrowth with fibrovascular tissue by 6 weeks and by 12 weeks the scaffold had induced the formation of a layer of dense, regularly-oriented collagenous tissue which significantly increased the thickness of the native tendon. This new tissue was well-integrated into the host tissues at both the bone interface and along the length of the tendon. At 26 weeks the scaffold was completely absorbed leaving a stable layer of mature tendon-like tissue over the surface of the host tendon which was still present at 52 weeks. The use of a reconstituted collagen scaffold consistently increased the thickness of a rotator cuff tendon by inducing the formation of a well-integrated and mature tendon-like tissue.
KEY WORDS: tendon, collagen scaffold, rotator cuff, histology, sheep.

\section{Introduction}

Partial-thickness tears of the supraspinatus tendon comprise a complex and significant pathological process that is increasing in prevalence as the population ages ${ }^{1-3}$. Unfortunately, there is no consensus as to the most appropriate treatment of partial-thickness, articular surface tears of the rotator cuff and a variety of treatments have been advocated ranging from strengthening exercises, to debridement, to acromioplasty, to in situ repair, to completing the tear so as to allow for a conventional surgical repair ${ }^{1,4,5}$. In a cohort of non-operated partial-thickness tear patients, $80 \%$ of partial-thickness rotator cuff tears demonstrated progression of the tear over a two-year period, with $28 \%$ of these patients progressing to a full-thickness tear ${ }^{6}$. Surgical repair has classically been advocated for partial-thickness tears involving $50 \%$ or more of the tendon thickness, however, these repairs are not always successful ${ }^{1}$.

Partial-thickness tears of the rotator cuff have been theorized to give rise to non-physiologic tension on the remaining tendon fibers ${ }^{7}$. Several in vitro studies have implicated the significant increase in intra-tendinous strain following partial-thickness tears of the supraspinatus tendon as a predisposing factor for tear propagation ${ }^{8-10}$. In addition, this increased intratendinous strain could further contribute to the compromised healing environment of these partial-thickness defects ${ }^{1}$. The ability to rapidly increase the thickness and resulting cross-sectional area of an injured tendon through the natural regeneration of additional tendon-like tissue could, theoretically, decrease the stress (and resulting strain) experienced by the injured tendon. This could not only lessen the chance for future tear propagation, but may also allow for a more biomechanically conducive environment for healing.

Therefore, it was the purpose of this study to characterize the ability of a highly porous reconstituted collagen scaffold of low tensile modulus to increase the thickness of an injured tendon through the induction and remodeling of additional tendinous tissue. It was hypothesized that a highly porous, biocompatible scaffold placed on the surface of a damaged infraspinatus tendon would induce a rapid and proliferative ingrowth of host tissue to increase the thickness of the tendon. It was also hypothesized that using scaffold with a low tensile modulus relative to the ten- 
don would create a strain environment that would encourage functional alignment of additional tendon-like tissue along the natural axis of tendon loading.

\section{Methods}

\section{Collagen Scaffold}

The reconstituted collagen scaffolds were made from highly-purified, type I collagen from bovine tendons using proprietary methods (Collagen Matrix, Inc., Oakland, New Jersey) ${ }^{11,12}$. The collagen fibers were processed to create a highly oriented and highly porous (85-90\% porosity) collagen scaffold with a DNA content of less than $50 \mathrm{ng} / \mathrm{mg}$. The scaffolds were freeze-dried after processing and rehydrated at the time of surgery. The rehydrated scaffold dimensions were approximately $15 \mathrm{~mm}$ wide, $30 \mathrm{~mm}$ long, and $2 \mathrm{~mm}$ thick (Fig. 1). The scaffold had a tensile modulus of approximately $6 \mathrm{MPa}$ when tested in its long dimension.

\section{Animal Procedures}

All animal procedures were approved by the Colorado State University Institutional Animal Care and Use Committee. Skeletally mature (>3.5 year-old) female sheep (Ovis aries) were used in this study. Twentythree sheep were randomly assigned to survival times of $6,12,26$, and 52 weeks. Preoperative analgesics and antibiotics were provided and under general anesthesia the infraspinatus tendon of the right shoulder was surgically exposed. The infraspinatus tendon was left attached to the humerus; however, in order to simulate a tendon injury, four $2 \mathrm{~mm}$-deep slits were made in the infraspinatus tendon along its entire length in the direction of the tendon fibers. Three bone tunnels were made distal to the insertion of the infraspinatus tendon for suturing the scaffold to bone. The scaffold (approximately the same width and length as the tendon) was rehydrated in physiologic saline and attached at one end to bone using $3 / 0$ braided polyester sutures that were passed through the bone tunnels. The free end of the scaffold was then sutured under slight tension to the infraspinatus tendon with interrupted $3 / 0$ braided polyester sutures (Fig. 2). The surgical site was lavaged with sterile saline prior to closure. Sham procedures were performed in 6 additional sheep, wherein all elements of the surgery were performed except that no scaffold was implanted. Three sham animals were evaluated at each of two time periods ( 6 and 12 weeks).

Following recovery from anesthesia, the animals were returned to indoor pens without immobilization and allowed unrestricted activity. Postoperative analgesics were provided to control pain. The sheep were closely monitored for any adverse effects (lethargy, lack of appetite, etc.) or signs of lameness. At the allocated survival times the sheep were humanely euthanized by an intravenous injection of sodium pento-
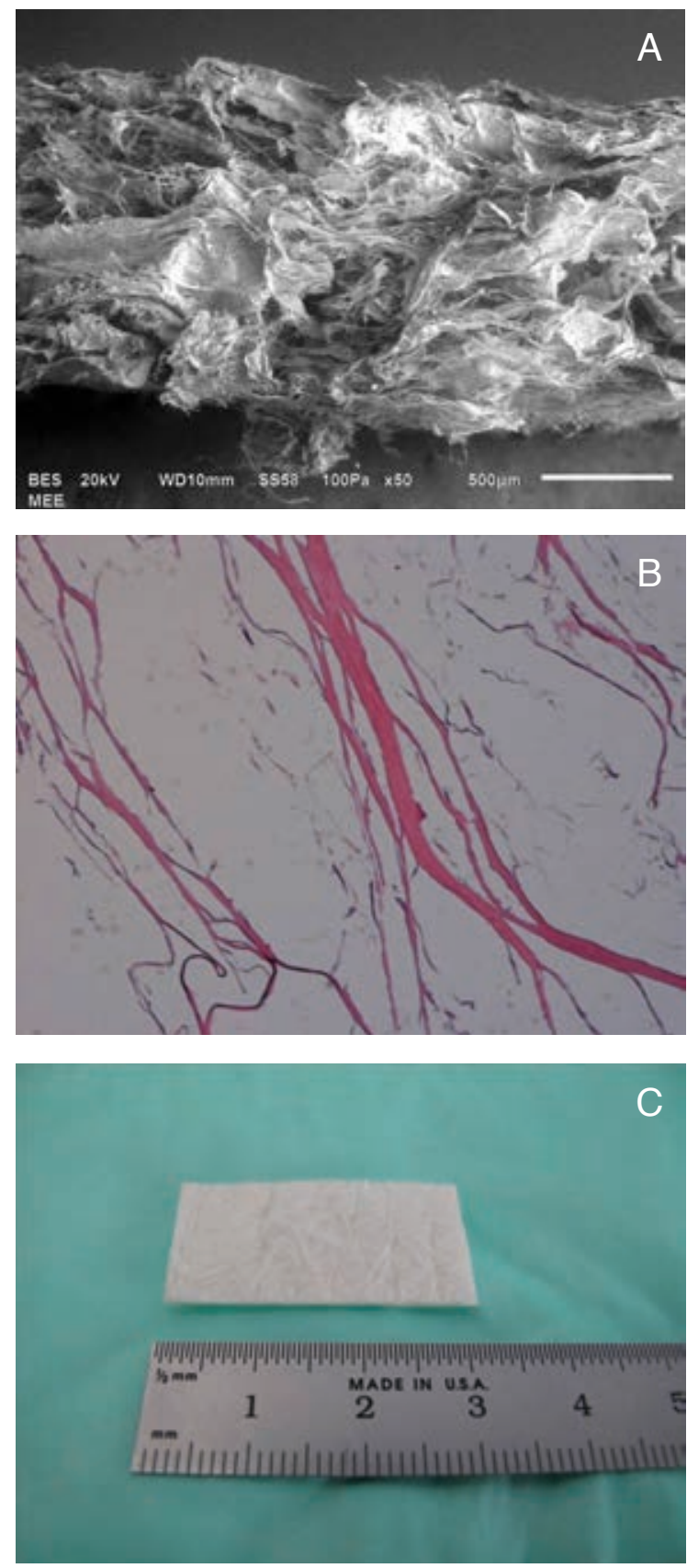

Figure 1. (A) Scanning electron micrograph of the crosssection of a cryofractured reconstituted collagen scaffold. Bar=500 microns, original magnification=50x. (B) Histological section of the collagen scaffold prior to implantation. $\mathrm{H} \& \mathrm{E}$, original magnification $=50 \mathrm{x}$. (C) Photograph of the collagen scaffold prior to implantation.

barbital. During necropsy the infraspinatus muscle was detached from the scapula and the proximal onethird of the humerus was harvested. Care was taken not to disturb the deltoid muscle immediately over the implant area. The bone-tendon-muscle complex, including the scaffold and a portion of the overlying deltoid muscle, was placed in $10 \%$ buffered formalin for fixation. 


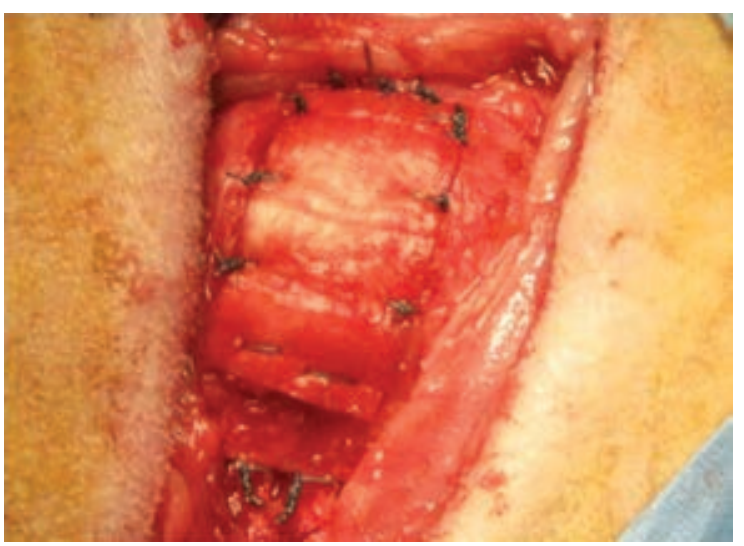

Figure 2. Operative view of the reconstituted collagen scaffold sutured to the superior surface of the infraspinatus tendon. Scaffold size $=15 \times 30 \mathrm{~mm}$.

\section{Histological Analysis}

In order to examine the entire bone-tendon-scaffold complex in situ, the formalin-fixed specimens were frozen at $-80^{\circ} \mathrm{C}$ and the entire complex bisected along the long axis of the infraspinatus tendon using a band saw. The bisected bone-tendon specimens were photographed and the digital images used to determine the thickness of the new tissue. The thickness of the new tissue overlying the infraspinatus tendon was measured at 3 locations and the average expressed as a percentage of infraspinatus tendon thickness of each animal. The bone-tendon-scaffold specimens were then decalcified in $5 \%$ nitric acid solution, embedded in paraffin, and 5 micron-thick sections cut and stained with hematoxylin and eosin (H\&E). The slides were analyzed using both transmitted and polarized light microscopy to evaluate host response, tissue ingrowth, collagen fiber alignment, and integration of new tissue with host bone and tendon.

\section{Results}

All of the sheep tolerated the surgery well and no postoperative pain or lameness was observed. One animal developed a superficial skin infection that progressed down into the shoulder joint. This animal was excluded from the study.

\section{Collagen Scaffold Group}

After 6 weeks of implantation the voids in the collagen scaffold were completely filled with proliferating fibrovascular tissue (Fig. 3). The fibers of the collagen scaffold were clearly evident and easily distinguished from the new tissue ingrowth. These observations were consistent in all specimens. There was no evidence of any inflammatory or foreign body reaction in any of the 6-week specimens. The mean thickness of the scaffold-induced tissue was $70.8 \% \pm$

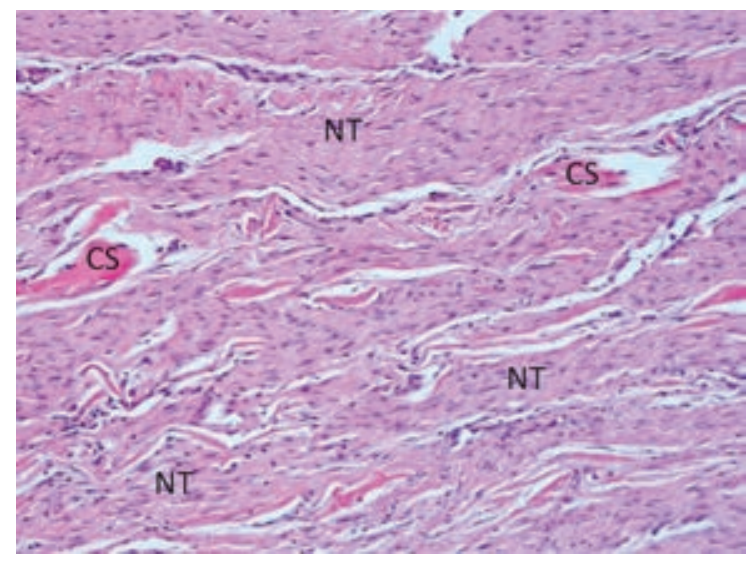

Figure 3. At 6 weeks after implantation the porosity of the collagen scaffold (CS) was fully ingrown with new fibrovascular tissue (fibroblasts, blood vessels, and matrix). NT=New Tissue. No inflammatory or foreign body reaction. $\mathrm{H} \& E$, original magnification $=100 x$.

$11.3 \%$ (std dev) of the thickness of the underlying infraspinatus tendon.

After 12 weeks of implantation a layer of new connective tissue overlying the superior surface of the infraspinatus tendon and its bony insertion was clearly visibly (Fig. 4A). The mean tissue thickness was $80.5 \% \pm$ $16 \%$ (std dev) of the thickness of the underlying infraspinatus tendon. Histologically, this new tissue was composed of fibroblasts and regularly-oriented collagen fibers (Fig. 4B) and was intimately attached to the underlying infraspinatus tendon (Figs. 4C, 4D). The fibers of the collagen scaffold were occasionally visible in the 12 -week sections. The scaffold-induced connective tissue demonstrated good integration into bone and there was evidence of Sharpey's fibers (Fig. 5). These observations were consistent in all specimens. There was no evidence of any inflammatory or foreign body reaction in any of the 12-week specimens.

After 26 weeks of implantation, the layer of new connective tissue overlying the infraspinatus tendon appeared more mature and regularly-oriented (Fig. 6). The mean tissue thickness was $91.5 \% \pm 0.6 \%$ (std dev) of the thickness of the underlying infraspinatus tendon. There was no evidence of any remaining collagen scaffold in the 26-week sections. The bony insertion of the new tissue demonstrated evidence of a fibrocartilagenous component, suggestive of a more normal direct insertion (Fig. 7). There was no evidence of any inflammatory or foreign body reaction in any of the 26-week specimens.

The histological response remained stable out to 52 weeks of implantation. The scaffold-induced tissue was less cellular and histologically resembled tendonlike (dense, regularly-oriented) connective tissue and was well-integrated with the underlying host tendon (Fig. 8). The mean tissue thickness was $86.1 \% \pm$ $13.6 \%$ (std dev) of the thickness of the underlying infraspinatus tendon. There was no evidence of any inflammatory or foreign body reaction in any of the 52week specimens. 

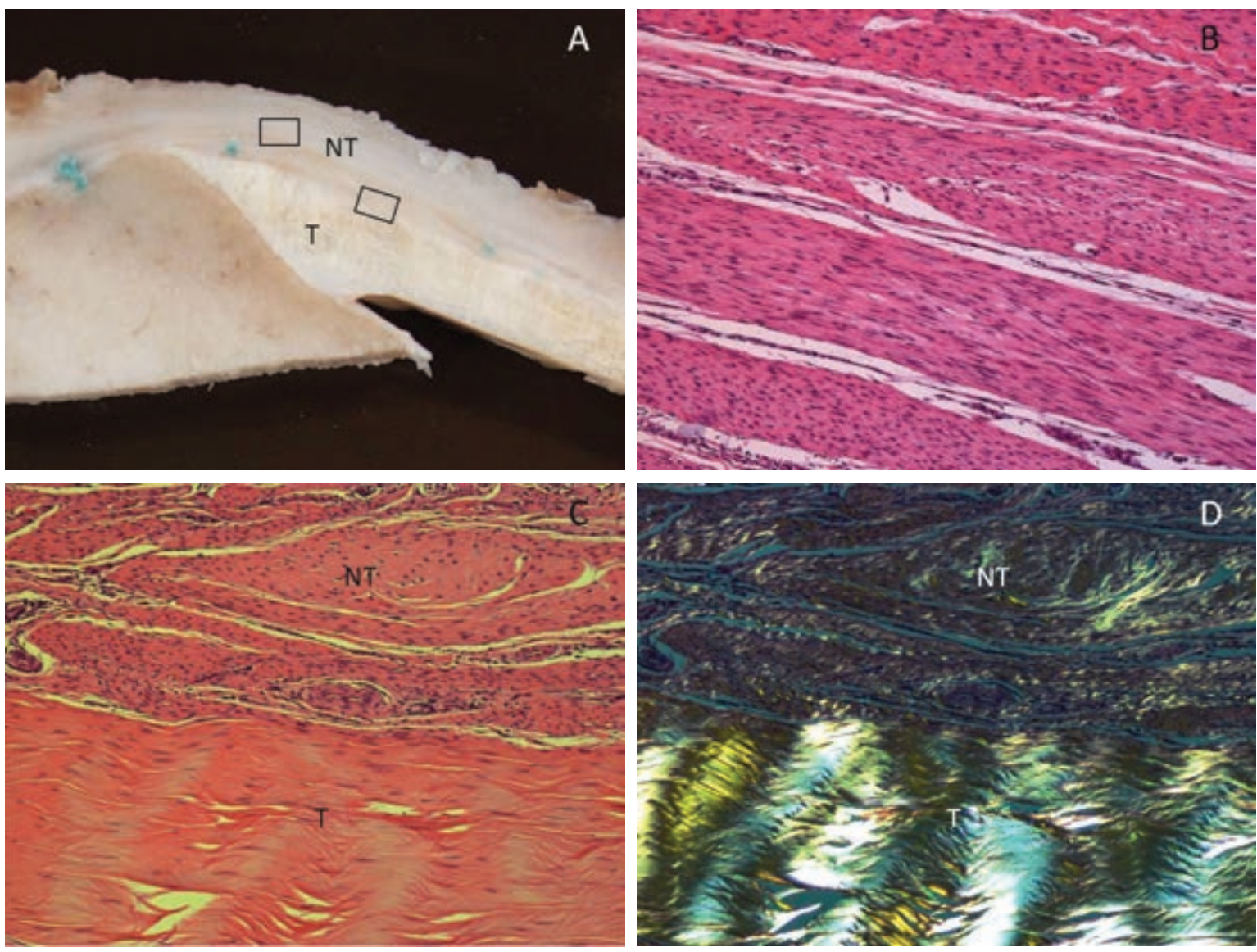

Figure 4. New tissue induced by the collagen scaffold at 12 weeks after implantation. (A) Gross photograph of the new tissue (NT) on top of the tendon (T). The squares indicate areas that are shown histologically in Panels B, C, and D. (B) Regularly-oriented collagen fibers in the area of the square on the left in Panel A. No inflammatory or foreign body reaction. H\&E, original magnification=100x. (C) Interface between the new tissue (NT) and the tendon (T) in the area of the square on the right in Panel A showing continuity of collagen fibers between the new tissue and the underlying tendon. No inflammatory or foreign body reaction. H\&E, original magnification=100x. (D) Polarized light image of Panel C.
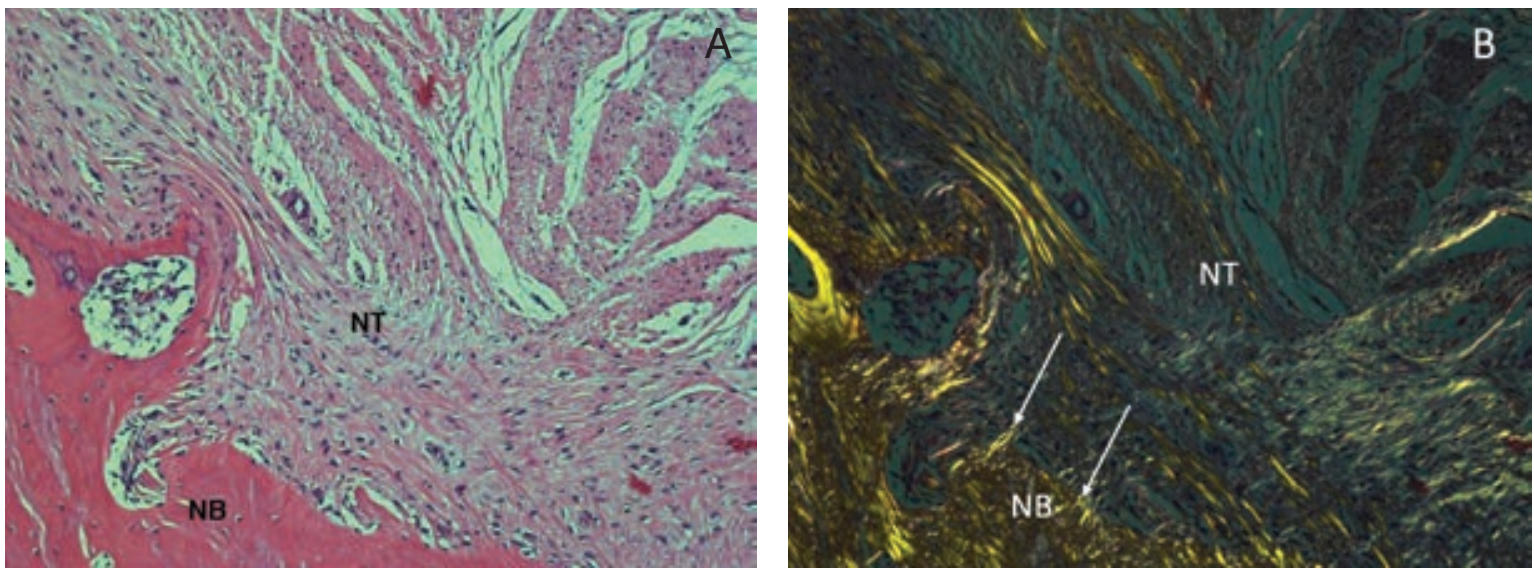

Figure 5. Interface of the new tissue with bone at 12 weeks after implantation. (A) Integration of the new tissue (NT) into the native bone (NB). No inflammatory or foreign body reaction. H\&E, original magnification=100x. (B) Polarized light image of Panel A. Arrows indicate Sharpey's fibers at the interface of the new tissue with the native bone.

\section{Sham Control Group}

The sham procedures, wherein all elements of the surgical procedure were performed except that no scaffold was implanted, demonstrated that, in the absence of the scaffold, there was no discernible induc- 
Tissue-engineered augmentation of a rotator cuff tendon using a reconstituted collagen scaffold: a histological evaluation in sheep

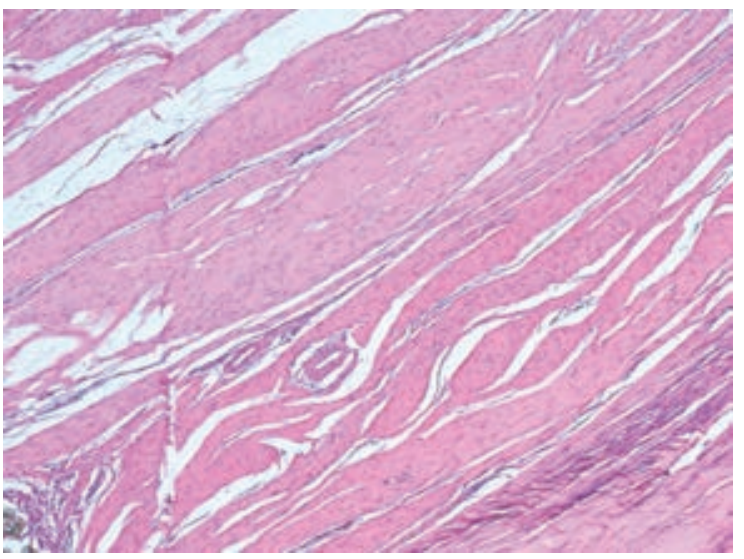

Figure 6. At 26 weeks after implantation the new tissue consisted of well-organized connective tissue. The collagen scaffold was completely absorbed by 26 weeks. No inflammatory or foreign body reaction. H\&E, original magnification $=50 \mathrm{x}$.
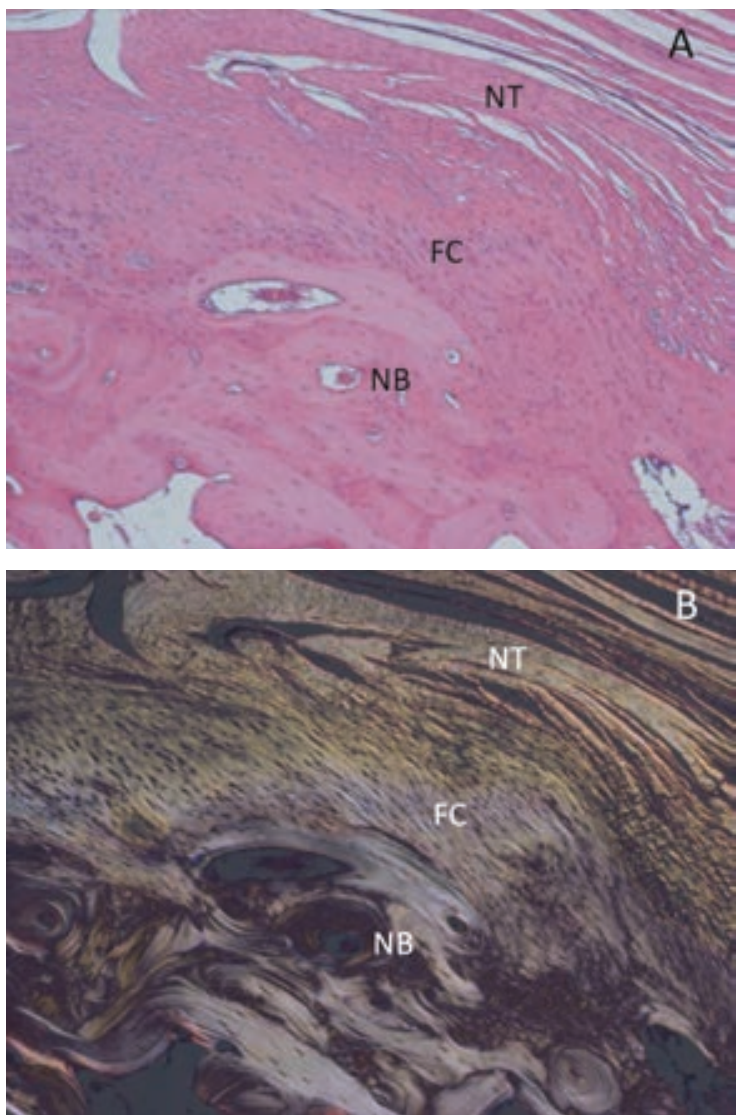

Figure 7. (A) At 26 weeks after implantation the new tissue (NT) was well-integrated with the native bone (NB) with a layer of fibrocartilage (FC) at the bone interface. No inflammatory or foreign body reaction. H\&E, original magnification=50x. (B) Polarized light image of Panel A.

tion of new tissue over the superior surface of the infraspinatus tendon at the two time periods examined (6 and 12 weeks).

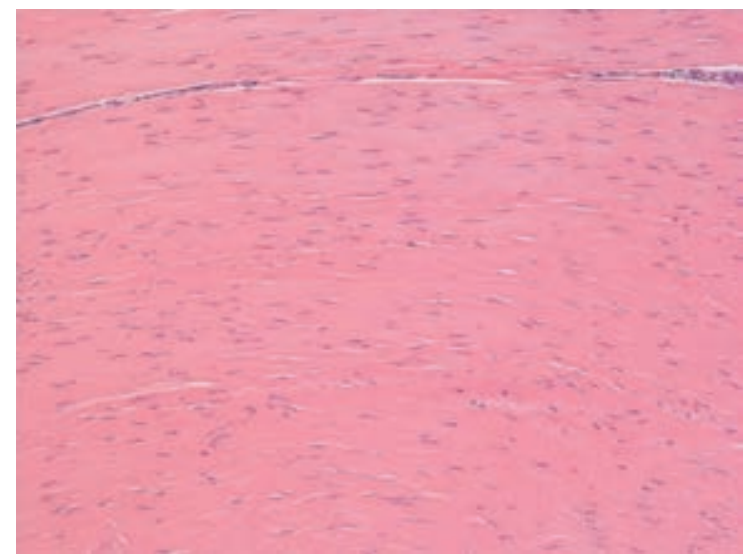

Figure 8. At 52 weeks after implantation the new tissue consisted of well-organized connective tissue that was well-integrated with the underlying tendon. No inflammatory or foreign body reaction. H\&E, original magnification $=100 x$.

\section{Discussion}

Collagen-based extracellular matrices have previously been advocated as an augmentation to the surgical re-attachment of large tears of the rotator cuff tendons ${ }^{13,14}$. The primary indication of these matrices is to provide supplemental support, protection, and reinforcement (i.e., augmentation) of repaired rotator cuff tendons ${ }^{13}$. However, variations in degradation rates, biocompatibility, and tissue-induction properties of currently available collagen-based scaffolds may affect the predictability of their biologic behavior ${ }^{14,15}$.

The results of the current study support the hypothesis that a highly porous, biocompatible, reconstituted collagen scaffold of low tensile modulus could consistently support the rapid ingrowth of host tissue and swiftly induce its progressive maturation into a thick layer of regularly-oriented connective tissue. The new tissue became integrated with the underlying infraspinatus tendon and was able to increase the thickness of the tendon by $80 \%$ at 12 weeks. This increase in mass was maintained over the next 9 months as the new tissue matured and became more tendon-like in its histological appearance. It is likely that the low $(6 \mathrm{MPa})$ modulus of the scaffold permitted sufficient deformation of the new tissue to allow its modulation from fibrovascular repair tissue to dense, regularly-oriented connective tissue. The initial mechanical properties of the collagen scaffold were an order of magnitude below that reported for the rotator cuff tendons of animals ${ }^{13}$ and humans ${ }^{16}$. The lower tensile modulus could be an important factor in the rapid orientation of the collagen fibers seen in the current study. Increased strain has been shown to induce collagen fiber alignment ${ }^{17}$ which relates to improved material properties (over less-aligned collagen fibers) ${ }^{17}$.

While mechanical loading is necessary for the functional remodeling of the newly synthesized tissue ${ }^{18}$, it is also required for the creation of a normal tendon- 
bone insertion ${ }^{19}$. In the current study, the scaffold-induced tissue appeared well-integrated into cortical bone by 12 weeks with evidence of Sharpey's fibers spanning the osseous attachment of the new tissue. By 26 weeks the new tissue demonstrated a fibrocartilagenous transition zone at its insertion site into bone. This is similar to the direct insertion site described for the bony attachment of rotator cuff tendons. The creation of a direct type of insertion site that allows gradations in structure, composition, and material properties does not always occur in surgical reattachment of the rotator cuff and this is thought to be responsible for the high incidence of failure of these repairs ${ }^{19,20}$.

The primary limitation of this research is that it was performed in healthy sheep with intact tendons. While sheep are considered appropriate animal models for studying the repair of full-thickness tears of rotator cuff tendons, partial-thickness tendon lesions in healthy animals can heal spontaneously ${ }^{21}$. Therefore, the premise that adding new regularly-oriented, connective tissue to a rotator cuff tendon with a partial-thickness tear could decrease the incidence of tear propagation or allow for a more biomechanically optimal healing environment for these partial-thickness tears cannot be accurately tested in healthy animals. However, the lack of any new tissue accretion in sham animals supports the inductive and conductive capabilities of the reconstituted collagen scaffold in generating new tendon-like tissue. Additionally, the current study did not examine the material or biochemical characteristics of the new tissue augmenting the infraspinatus tendon. While this limits our ability to fully characterize the new tissue as being a tendon, the histologic characteristics of the maturing tissue observed in this study (i.e. dense, regularly-oriented connective tissue) is similar to that of tendons ${ }^{22}$. However, the ultimate test of any regenerated tissue will be its ability to integrate and function within the host environment. Finally, although increasing the thickness of the infraspinatus tendon by $80 \%$ through the addition of collagenous tissue highly oriented along the axis of loading would likely decrease any applied stress (and ultimately strain) experienced by the tendon, additional studies are needed to confirm this supposition.

The results of this study demonstrate that a highly porous, low modulus scaffold made of highly-purified reconstituted collagen has the ability to induce the formation of new tendon-like tissue consisting of wellorganized collagen with fibers oriented in the direction of the load. The scaffold did not elicit an inflammatory response or foreign body reaction and was completely absorbed within 6 months after implantation. The new tissue induced by the scaffold was wellintegrated with the underlying rotator cuff tendon and demonstrated a fibrocartilagenous transition zone at its insertion into bone. The increased thickness of the infraspinatus tendon appeared stable throughout the 12-month follow-up of this study. These results suggest that the reconstituted collagen scaffold may have a role in preventing tear propagation in partialthickness tears of the supraspinatus tendon by in- creasing tendon thickness and decreasing intra-tendinous strain.

\section{Acknowledgement}

The authors thank Keri Gardner, LVT, MS, Kimberly Lebsock, BS, and Sheila Pelkey, AAS, CVT, for their technical support on this study.

\section{References}

1. Finnan RP, Crosby LA. Partial-thickness rotator cuff tears. J Shoulder Elbow Surg 2010; 19:609-616.

2. Gartsman GM, Milne JC. Articular surface partial-thickness rotator cuff tears. J Shoulder Elbow Surg 1995; 4:409-415.

3. Sher JS, Uribe JW, Posada A, Murphy BJ, Zlatkin MB. Abnormal findings on magnetic resonance images of asymptomatic shoulders. J Bone Joint Surg Am 1995; 77:10-15.

4. Franceschi F, Papalia R, Del Buono A, Maffulli N, Denaro V. Repair of partial tears of the rotator cuff. Sports Med Arthrosc 2011; 19:401-408.

5. Wang AW, Bauer S, Goonatillake M, Breidahl W, Zheng MH. Autologous tenocyte implantation, a novel treatment for partial-thickness rotator cuff tear and tendinopathy in an elite athlete. BMJ Case Rep Jan 11 2013, doi: 10.1136/bcr-2012007899.

6. Yamanaka K, Matsumoto T. The joint side tear of the rotator cuff: A followup study by arthrography. Clin Orthop Rel Res 1994; 304:68-73.

7. Wolff AB, Sethi P, Sutton KM, Covey AS, Magit DP, Medvecky M. Partial-thickness rotator cuff tears. J Am Acad Orthop Surg 2006; 14:715-725.

8. Bey MJ, Ramsey ML, Soslowsky LJ. Intratendinous strain fields of the supraspinatus tendon: Effect of a surgically created articular-surface rotator cuff tear. J Shoulder Elbow Surg 2002; 11:562-569.

9. Reilly P, Amis AA, Wallace AL, Emery RJ. Supraspinatus tears: Propagation and strain alteration. J Shoulder Elbow Surg 2003; 12:134-138.

10. Yang S, Park HS, Flores S, Levin SD, Makhsous M, Lin F, Koh J, Nuber G, Zhang LQ. Biomechanical analysis of bursal-sided partial-thickness rotator cuff tears. J Shoulder Elbow Surg 2009; 18:379-385.

11. Li ST, Yuen D. Oriented biopolymeric membrane. United States of America Patent Number 6,391,333; May 21, 2002.

12. Li ST, Yuen D. Oriented biopolymeric membrane. United States of America Patent Number 6,599,524; July 29, 2003.

13. Derwin KA, Baker AR, Spragg RK, Leigh DR, lannotti JP. Commercial extracellular matrix scaffolds for rotator cuff tendon repair: Biomechanical, biochemical, and cellular properties. J Bone Joint Surg Am 2006; 88:2665-2672.

14. Longo UG, Lamberti A, Maffulli N, Denaro V. Tendon augmentation grafts: A systematic review. Br Med Bull 2010; 94:165-188.

15. Reverchon E, Baldino L, Cardea S, DeMarco I. Biodegradable synthetic scaffolds for tendon regeneration. Muscle, Ligament, Tendon Journal 2012; 2:181-186.

16. Itoi E, Berglund L, Grabowski J, Schultz F, Growney E, Morrey $\mathrm{B}, \mathrm{An} \mathrm{KN}$. Tensile properties of the supraspinatus tendon. J Orthop Res 1995; 13:578-584.

17. Daniels F, ter Haar Romeny BM, Rubbens M, van Assen $\mathrm{H}$. Quantification of collagen orientation in 3D engineered tissue. IFMBE Proceedings 2007; 15:282-286. 
Tissue-engineered augmentation of a rotator cuff tendon using a reconstituted collagen scaffold: a histological evaluation in sheep

18. Giai Via A, DeCupis M, Spoliti M, Oliva F. Clinical and biological aspects of rotator cuff tears. Muscle, Ligament, Tendon Journal 2013; 3:70-79.

19. Thomopoulos S, Genin GM, Galatz LM. The development and morphogenesis of the tendon-to-bone insertion: What development can teach us about healing. J Musculoskel Neuronal Interact 2010; 10:35-45.
20. Wildemann B, Klatte F. Biological aspects of rotator cuff healing. Muscle, Ligament, Tendon Journal 2011; 1:161-168.

21. Turner AS. Experiences with sheep as an animal model for shoulder surgery: Strengths and shortcomings. J Shoulder Elbow Surg 2007; 16:158S-163S.

22. Ross MH, Pawlina W: Histology A text and atlas. 5th edition, 2006, Lippincott Williams \& Wilkins, Baltimore, MD. Pp 178-179. 